\title{
Disulfiram sensitizes pituitary adenoma cells to temozolomide by regulating 06-methylguanine-DNA methyltransferase expression
}

\author{
YACHAO ZHAO ${ }^{1}$, ZHENG XIAO $^{1}$, WENNA CHEN $^{2}$, JINSHENG YANG $^{1}$, TAO LI $^{1}$ and BO FAN ${ }^{1}$ \\ Departments of ${ }^{1}$ Neurosurgery and ${ }^{2}$ Neurology, The First Affiliated Hospital of Henan University of Science and Technology, \\ Luoyang, Henan 471003, P.R. China
}

Received July 17, 2014; Accepted March 24, 2015

DOI: $10.3892 / \mathrm{mmr} .2015 .3664$

\begin{abstract}
O6-methylguanine-DNA methyltransferase (MGMT) activity is responsible for temozolomide (TMZ) resistance in patients harboring aggressive pituitary adenomas. Recently, disulfiram (DSF) has been shown to induce the loss of MGMT protein and increase TMZ efficacy in glioblastoma cells, while CD133+ nestin+ cells isolated from the cell population have been implicated as pituitary adenoma stem-like cells. However, whether DSF is able to potentiate the cytotoxic effects of TMZ on human pituitary adenoma cells has not been investigated to date. In the present study, CD133+ nestin+ phenotype cells were isolated from primary cultured human pituitary adenoma cells using microbeads. It was found that DSF reduced MGMT protein expression and sensitized human pituitary adenoma cells and stem-like cells to TMZ in vitro, while the proteasome inhibitor PS-341 abrogated the inhibitory effect of DSF on MGMT in vitro. The sensitizing effect of DSF was also verified in primary cultured human pituitary adenoma cells in vivo. The results of the present study suggested that DSF can increase the efficacy of the anti-tumor effect of TMZ on human pituitary adenoma cells and CD133+ nestin+ stem like cells via the ubiquitin-proteasomal MGMT protein elimination route. DSF combined with TMZ may be an effective therapeutic strategy against aggressive pituitary adenomas.
\end{abstract}

\section{Introduction}

Pituitary adenomas are common intracranial tumors, with an incidence of $15 \%$ in intracranial neoplasms (1-4). Clinically, aggressive pituitary adenomas are observed in up to $33 \%$ of pituitary adenomas $(5,6)$. Despite surgery combined with radiation- and chemotherapy, patients with aggressive pitu-

Correspondence to: Professor Bo Fan, Department of Neurosurgery, The First Affiliated Hospital of Henan University of Science and Technology, 24 Jinghua Road, Luoyang, Henan 471003, P.R. China E-mail: bofanly@163.com

Key words: pituitary adenoma, disulfiram, O6-methylguanine-DNA methyltransferase, temozolomide itary adenomas still have a poor prognosis due to aggressive tumor characteristics and the lack of effective therapies, which remains an enormous therapeutic challenge (7-10).

Temozolomide (TMZ) is currently considered the most promising chemotherapeutic drug in the treatment of aggressive pituitary adenomas resistant to conventional standard therapies (11-15). However, the efficacy of TMZ treatment in aggressive pituitary adenoma patients in clinical trials varies largely $(12,16-18)$. Numerous patients who were treated at our clinic were classified as TMZ nonresponders. Furthermore, patients displaying an initial response to $\mathrm{TMZ}$ treatment may present with tumor relapse after a certain number of treatment cycles due to increasing TMZ resistance $(16,17)$. These treatment shortfalls justify an urgent requirement for novel therapeutic approaches to improve the TMZ efficacy in pituitary adenomas.

In a previous study by our group using primary cultured human pituitary adenoma cells, experimental evidence suggested that O6-methylguanine-DNA methyltransferase (MGMT) is the key factor responsible for chemoresistance to TMZ, and 2-methoxyestradiol (2ME) may be applied as a mediator of TMZ resistance via the downregulation of MGMT expression (19). Besides 2ME, disulfiram (DSF), having the ability to cross the blood-brain barrier, has recently been reported to be a potential inhibitor of MGMT in glioblastoma cells (20). Whether DSF can inhibit MGMT expression and improve the efficacy of $\mathrm{TMZ}$ in human pituitary adenomas has remained elusive. In the present study, the effect of DSF on MGMT protein expression levels and on the anti-tumor effect of TMZ on primary cultured human pituitary adenoma cells and isolated CD133+ nestin+ phenotype stem like cells was investigated in vitro. Furthermore, the sensitizing effect was also assessed in differentiated human pituitary adenoma cells in vivo.

\section{Materials and methods}

Patients and samples. The present study was approved by the Research Ethics Committee of the Henan University of Science and Technology (Luoyang, China). Prior informed consent was obtained from the patients harboring huge aggressive pituitary adenomas. The aggressive pituitary adenoma characteristic of the patients was classified based on magnetic resonance imaging (MRI) results and history of illness prior 
to surgery, which showed pituitary adenomas of large size, massive invasion of surrounding anatomical structures and rapid growth indicated by comparison with their previous MRI scans. Between June 2012 and January 2014, after surgery for patients harboring huge aggressive pituitary adenomas, the pituitary adenoma fragments were divided into three parts; one part was sent to the Pathology Department, one part was investigated for MGMT and Ki67 using western blot analysis, and another part was primarily cultured for further possible analysis. Two MGMT-negative human pituitary-null cell adenoma tissues were identified and used as controls. Whenever western blot analysis of tumor tissues showed that the pituitary adenoma was strongly MGMT-positive, further primary culture or tumor implantation in nude mice was performed. Twelve strongly MGMT-positive pituitary adenoma samples and two MGMT-negative pituitary adenoma samples were successfully primarily cultured or transplanted into nude mice in the present study.

Primary culture of pituitary adenoma tissue. The human pituitary adenoma tissues was processed according to the standard protocols as described in a previous study by our group (19) directly following surgery. The tissue was enzymatically digested using the Human Tumor Dissociation Kit (\#130-095929; Miltenyi Biotec, Bergisch Gladbach, Germany) with the gentle MACS Dissociator (\#130-093-235; Miltenyi Biotec). After dissociation, the sample was filtered using anti-human Fibroblast MicroBeads (\#130-050-601; Miltenyi Biotec). The cells were then counted and cultured in $16-\left(10^{6}\right.$ cells/well $)$ or 96 -well plates $\left(10^{4}\right.$ cells/well) in complete DMEM/F12 medium (Gibco Life Technologies, Carlsbad, CA, USA) with $2 \mathrm{mM}$ glutamine (Gibco Life Technologies), 15\% horse serum (Gibco Life Technologies) and 2.5\% fetal bovine serum (Gibco Life Technologies) for primary culture, with reference to the study by Xu et al (21).

Isolation for pituitary adenoma stem-like cells. The isolation of stem-like cells was performed as described in a previous study by our group (22). In brief, the human pituitary adenoma cells in primary culture were dispersed, counted and suspended $\left(10^{8}\right.$ cells) in magnetic micro beads buffer (\#130-091-376; Miltenyi Biotec) in a final volume of $600 \mu \mathrm{l}$. The suspension was added in FcR blocking reagent (\#130-059-901; $200 \mu \mathrm{l}$ ) and $200 \mu \mathrm{l}$ CD133 MACS micro beads (\#130-050-801; Miltenyi Biotec). The suspension was then mixed and incubated for $15 \mathrm{~min}$ at $4^{\circ} \mathrm{C}$. Using a MACS Separator (\#130-042-602; Miltenyi Biotec), the suspension was passed through a CD133 column, the column was placed on a collection tube, and the CD133-positive cells were obtained. Then, the resuspended cells were cultured with mouse monoclonal anti-nestin antibody (\#196908; R\&D Systems, Inc., Westerville, OH, USA) for $30 \mathrm{~min}$ at $4^{\circ} \mathrm{C}$ and were washed with phosphate-buffered saline (PBS; Wuhan Boster Biological Technology, Ltd., Wuhan, China). The cells were then mixed with anti-mouse IgG MicroBeads (\#130-048-401; Mitenyi Biotec) and proceeded to magnetic separation as described above. In reference to the protocol of a previous study by $\mathrm{Xu}$ et al (21) for primary cultural pituitary adenoma cells prior to isolation, the cells were cultured in DMEM/F12 (1:1; Gibco Life Technologies), B27 (1X; Gibco
Life Technologies), penicillin/streptomycin $(200 \mathrm{U} / \mathrm{ml}$; Invitrogen Life Technologies, Carlsbad, CA, USA), fungizone (250 ng/ml; Peprotech, Inc., Rocky Hill, NJ, USA), epidermal growth factor $(20 \mathrm{ng} / \mathrm{ml}$; Peprotech, Inc.) and basic fibroblast growth factor $(20 \mathrm{ng} / \mathrm{ml}$; Peprotech, Inc.) prior to and after isolation in order to avoid cell differentiation.

Cell viability assay, western blot analysis, mitotic catastrophe assessment, flow cytometry, bromodeoxyuridine (BrdU) and terminal deoxynucleotidyl transferase dUTP nick end labeling (TUNEL) assay. In the present study, the protocols and the reagents of the cell counting kit (CCK) 8 cell viability assay, western blot analysis, mitotic catastrophe assessment and flow cytometry were identical to those described in previous studies by our group (23-27). For the CCK8 assay, the Cell Counting Kit (CCK-8/WST-8) was used (AR1160-500; Wuhan Boster Biological Technology, Ltd.). The BrdU assay was conducted using the BrdU Cell Proliferation Assay kit (\#551321; BD Biosciences, La Jolla, CA, USA). Mice were injected intraperitoneally with $200 \mu \mathrm{l}(3 \mathrm{mg} / \mathrm{ml})$ BrdU solution $2 \mathrm{~h}$ prior to scarification. Subsequently, cellular incorporation of BrdU was detected by immunohistochemistry using antiBrdU specific antibodies. The TUNEL assay was performed using the ApopTag Peroxidase In situ Apoptosis Detection kit (\#S7100; Merck Millipore, Darmstadt, Germany). The BrdU and TUNEL assays were performed following the manufacturer's instructions. In brief, for the CCK8 assay, DSF (T1132, Sigma-Aldrich, St. Louis, MO, USA) and TMZ (T2577; Sigma-Aldrich) were added at various concentrations to the primary culture of human pituitary adenoma cells for $24 \mathrm{~h}$, the CCK 8 assay reagent was added to each well of the 96 -well plate, followed by $1 \mathrm{~h}$ of incubation at $37^{\circ} \mathrm{C}$. Absorbance was read at $450 \mathrm{~nm}$ using the Thermo Multiskan Ascent plate reader (Thermo Fisher Scientific, Rockford, IL, USA). For mitotic catastrophe assessment, the cells were fixed with cold methanol $\left(4^{\circ} \mathrm{C}\right.$; Maixin Biotech Co., Fuzhou, China) and stained with DAPI for chromosome analysis under an Olympus BX41 fluorescence microscope (Olympus Corp., Tokyo, Japan). Mitotic catastrophe is defined as the presence of $\geq 2$ nuclear lobes within a single cell. For western blot analysis, equal aliquots $(30 \mu \mathrm{g})$ of protein were separated using 10-12\% SDS-PAGE gel and transferred to nitrocellulose membranes (EMD Millipore, Shanghai, China). The membranes were incubated with relevant primary antibodies overnight at $4^{\circ} \mathrm{C}$, the following antibodies were used: Rabbit polyclonal anti-MGMT antibody (FL-207) (1:1,000; sc-28241, Santa Cruz Biotechnology, Inc., Dallas, TX, USA), mouse monoclonal anti- $\gamma \mathrm{H} 2 \mathrm{AX}$ antibody (1:500; JBW 301, Upstate Biotechnology, Milford, MA, USA), rabbit polyclonal anti-Rad51 (H-92) antibody (1:1,000; sc-8349, Santa Cruz Biotechnology, Inc.), mouse monoclonal anti-GAPDH (A-3) antibody (1:3,000; sc-137179, Santa Cruz Biotechnology, Inc.). Subsequently, the membranes were incubated in horseradish peroxidase-conjugated secondary antibody (goat anti-mouse IgG; 1:5,000; \#31431; Thermo Fisher Scientific) and goat anti-rabbit IgG (1:5,000; \#31466; Thermo Fisher Scientific) for $1.5 \mathrm{~h}$ at room temperature. The membranes were then treated using Pierce ECL Western Blotting Substrate (Thermo Fisher Scientific) and visualized using a BioRad Universal 
A

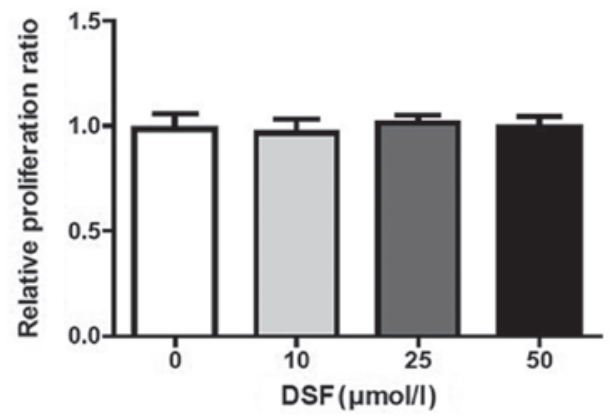

B

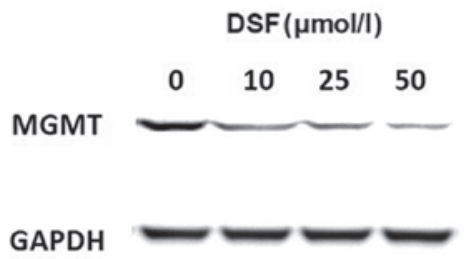

Figure 1. (A) Cell counting kit 8 assay showed that DSF alone displayed no obvious effect on the proliferation of human pituitary adenoma cells. Values expressed as the mean \pm standard deviation. (B) Effect of DSF on the MGMT protein expression levels in human pituitary adenoma cells. As shown in the western blot, DSF inhibited MGMT protein expression levels in a dose-dependent manner. GAPDH served as the loading control. MGMT, O6-methylguanine-DNA methyltransferase; DSF, disulfiram.

Hood II Imager (Bio-Rad Laboratories, Inc., Kenilworth, NJ, USA). For surface marker identification, the expression of the CD133 and nestin markers in cells was determined by flow cytometry after surface staining with anti-human mouse monoclonal CD133/1(AC133)-PE (\#130-080-801; Mitenyi Biotec) and mouse monoclonal anti-nestin (Ab81216; Abcam, Cambridge, MA, USA) antibodies. The cells were fixed with 4\% paraformaldehyde (10 min; Solarbio, Beijing, China) and then permeabilized with $0.1 \%$ phosphate-buffered saline (PBS)-Tween (Wuhan Boster Biological Technology, Ltd.) for 20 min. The cells were then incubated in 1x PBS/10\% normal goat serum (Wuhan Boster Biological Technology, Ltd.)/0.3 M glycine (Solarbio) to block non-specific protein-protein interactions followed by the antibody (1:200 dilution) for $30 \mathrm{~min}$ at $22^{\circ} \mathrm{C}$. The secondary antibody used was DyLight488 goat anti-mouse IgG $(\mathrm{H}+\mathrm{L})(\mathrm{ab96879}$; Abcam) at 1:500 dilution for $30 \mathrm{~min}$ at $22^{\circ} \mathrm{C}$. Flow cytometry was performed on a FACSCanto II (BD Biosciences) and analyzed using BD FCSDiva software, version 6 (BD Biosciences) and FCS Express 4 software (DeNovo Software, Glendale, CA, USA). For apoptosis analysis, the cells were suspended in Annexin V-fluorescein isothiocyanate (FITC) binding buffer (195 $\mu \mathrm{l})$ and Annexin V-FITC (5 $\mu \mathrm{l})$ in the dark for $10 \mathrm{~min}$. The cells were centrifuged at $1,000 \mathrm{x} \mathrm{g} / \mathrm{min}$ for $5 \mathrm{~min}$ and were suspended in binding buffer $(190 \mu \mathrm{l})$ and $10 \mu \mathrm{l}$ propidium iodide solution on ice in the dark for flow cytometric analysis.

Pituitary adenoma xenografts. Experiments on mice were conducted in line with protocols from the Institutional Animal Care and Use Committee and were approved by the Research Ethics Committee of the Henan University of Science and Technology (Luoyang, China). In the current study, 3-4 weeks old male BALB/c nude mice weighing 18-22 g were purchased from Shanghai Animal Center (Shanghai, China) and were housed five per cage in a specific pathogen-free (SPF) environment. The mice were kept on a 12-hour reversed light/dark cycle, the temperature was maintained at $25^{\circ} \mathrm{C}$, they were fed with SPF pellet diet (Beijing Huafukang Company, Beijing, China) and water was supplied. As described in previous studies by our group (23-25,27), in order to increase the tumor formation rate, the tissues were dissociated and then implanted subcutaneously into the three nude mice. Once the tumors were formed after two weeks, the mice were sacrificed by cervical dislocation and tumors from nude mice were dissociated and equally implanted subcutaneously into the lower rear flank of twelve six-week-old nude mice again. After the tumors had formed (following two weeks), the mice were injected with saline, TMZ (3 mg/kg), DSF $(50 \mathrm{mg} / \mathrm{kg})$ or TMZ (3 mg/kg) plus DSF (50 mg/kg) for five days (each group, $n=3$ ). Tumors were then harvested for further protein and histology analysis.

Statistical analysis. Statistical analyses were conducted using SPSS 13.0 (SPSS, Inc., Chicago, IL, USA). Values are expressed as the mean \pm standard deviation. Results were assessed using the Student's t-test and analysis of variance. $\mathrm{P}<0.05$ was considered to indicate a statistically significant difference between values.

\section{Results}

DSF decreases MGMT protein expression in human pituitary adenoma cells in primary culture. DSF has recently been shown to induce loss of MGMT protein in T98G and UW228 MGMT-proficient human glioblastoma cells (20); however, whether DSF can also downregulate the MGMT protein expression levels in human primary cultural pituitary adenoma cells has not been studied. Due to the lack of any well-established human pituitary adenoma cell line, human pituitary adenoma cells were obtained via primary culture of human pituitary adenoma tissue. DSF was added at various concentrations $(0,10,25$ and $50 \mu \mathrm{mol} / \mathrm{l})$ to the primary culture of human pituitary adenoma cells for $24 \mathrm{~h}$, followed by CCK8 assay and western blot analysis. As shown in Fig. 1A, the CCK8 assay showed that DSF alone in serum-free medium had no significant effect on the viability of the human pituitary adenoma cells, even at a concentration of $50 \mu \mathrm{mol} / \mathrm{l}(\mathrm{P}=0.835)$. However, DSF treatment significantly inhibited the MGMT protein expression levels in human primary cultural pituitary adenoma cells (Fig. 1B).

DSF inhibits protein expression of MGMT in putative pituitary adenoma stem-like cells. Recently, putative pituitary adenoma stem-like cells were isolated from the primary culture of human pituitary adenomas (21). The CD133- and nestin-expressing pituitary adenoma cells isolated from primary culture in the present study were capable of self-renewal and multipotent differentiation 
A

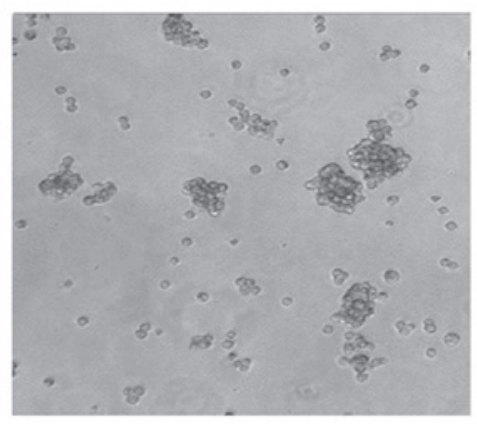

C
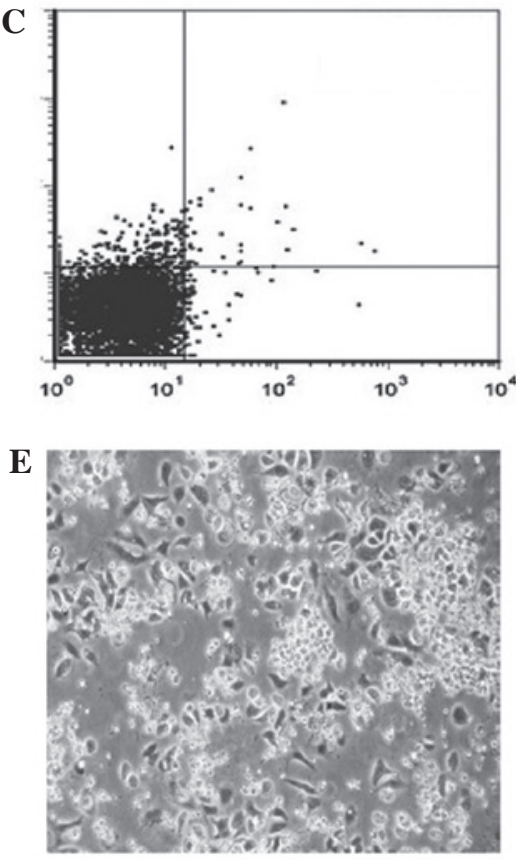

B
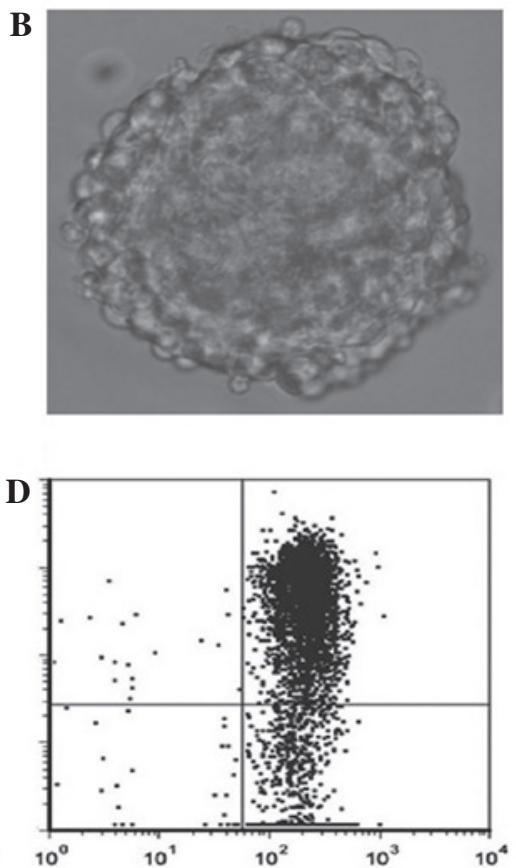

$\mathbf{F}$

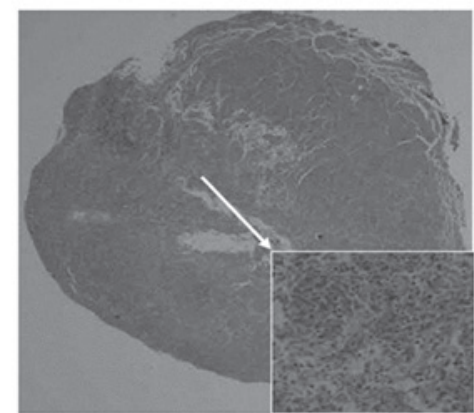

G Con Con DSF DSF

MGMT

GAPDH

Figure 2. Culture and characterization of the CD133+ nestin+ cell population of the human pituitary adenoma cells. (A) Culture of isolated CD133+ nestin+ cells growing as non-adherent spheres (magnification, $\mathrm{x} 40$ ). (B) Spheroids of CD133+ nestin+ cells (magnification, x400). (C) Representative cytometric dot-plots of CD133- and nestin-positive cells among human pituitary adenoma cells, displayed by the flow cytometric cell sorting using mouse monoclonal CD133 antibody and mouse monoclonal nestin antibody. (D) Representative cytometric dot-plots of CD133- and nestin-positive cells in isolated CD133+ nestin+ stem like cells, displayed by the flow cytometric cell sorting using mouse monoclonal CD133 antibody and mouse monoclonal nestin antibody. (E) Differentiation capacity of the isolated CD133+ nestin+ cells (magnification, x100). (F) High tumorigenic ability of CD133+ nestin+ cells. Hematoxylin and eosin staining of tumors generated from 10,000 CD133+ nestin+ cells in nude mice (magnification, $\mathrm{x} 10$ and x100). (G) As shown in the western blot, DSF (50 $\mu \mathrm{mol} / 1$ )inhibited MGMT protein expression in isolated CD133+ nestin+ cells. GAPDH served as the loading control. Con, control; MGMT, O6-methylguanine-DNA methyltransferase; DSF, disulfiram.

in vitro as well as initiation of serially transplantable pituitary tumors in vivo (21). The above evidence supported, at least to a certain extent, the tumor stemness of the isolated CD133+ nestin+ pituitary adenoma cells.

In previous studies by our group, cancer stem cells were isolated using magnetic microbeads (26). Furthermore, human pituitary adenoma cells from adenoma fragments were primarily cultured for studying the role of MGMT in human pituitary adenomas (27). Based on these previous studies, the present study also established primary cultures of human pituitary adenoma samples and isolated seem- ingly pituitary adenoma stem-like cells using CD133- and nestin-specific magnetic microbeads followed by flow cytometric cell sorting. As shown in Fig. 2C and D, the percentages of the CD133+ nestin+ cell sub-populations in the total pituitary adenoma cells in primary culture and isolated CD133+ nestin+ stem-like cells were $0.31 \pm 0.14 \%$ and $52.13 \pm 15.13 \%$, respectively. Although the pituitary adenoma stem-like cell phenotype has not been completely defined, the CD133+ nestin + pituitary adenoma cells in primary culture indeed generated spheres for several passages in the present study (Fig. 2A and B), indicating the self-renewal ability 
A

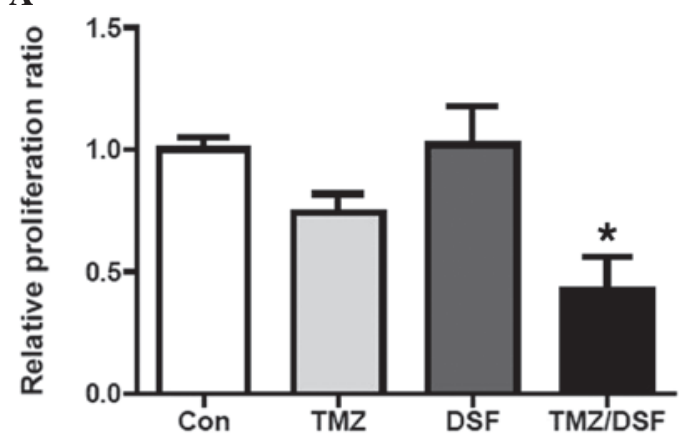

B

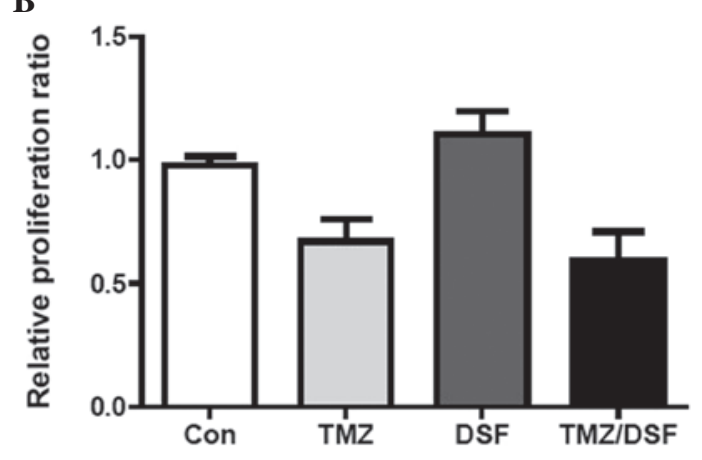

C

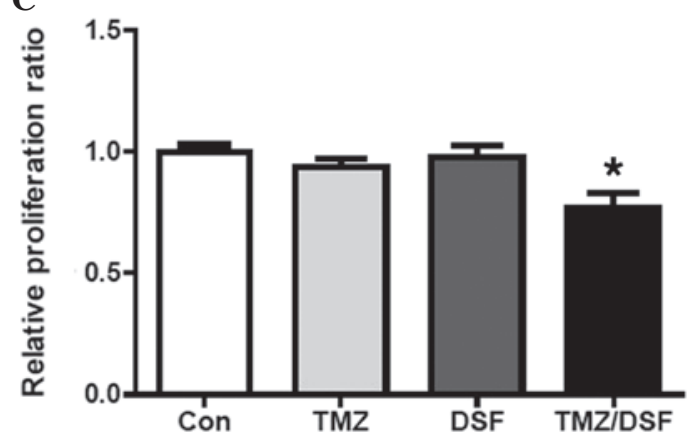

Figure 3. (A) CCK8 assay showed that DSF sensitized MGMT-proficient human pituitary adenoma cells to TMZ, increasing its anti-proliferative effects in vitro. (B) CCK8 assay showed that DSF did not sensitize MGMT-deficient human pituitary adenoma cells to TMZ in vitro. (C) CCK8 assay showed that DSF sensitized MGMT-proficient isolated CD133+ nestin+ human pituitary adenoma stem like cells to TMZ in vitro. Values are expressed as the mean \pm standard deviation from three independent experiments; ${ }^{*} \mathrm{P}<0.05$, vs. TMZ group. MGMT, O6-methylguanine-DNA methyltransferase; DSF, disulfiram; CCK, cell counting kit; TMZ, temozolomide.

of these cells. The cells from spheres can also differentiate into cells with a morphology similar to that of human pituitary adenoma cells (Fig. 2E). Moreover, a small number of cells $(10,000)$ that were injected intracranially and incubated for 6 weeks, formed xenograft tumors in the right hemisphere of nude mice (Fig. 2F), suggesting the differentiation capacity and high tumorigenic ability of the CD133+ nestin+ pituitary adenoma stem-like cells.

Since DSF was shown to inhibit MGMT in differentiated pituitary adenoma cells, the present study assessed whether DSF can also downregulate the MGMT protein expression levels in CD133+ nestin+ pituitary adenoma stem-like cells. As shown in Fig. 2G, western blot analysis showed that DSF $(50 \mu \mathrm{mol} / \mathrm{l})$ treatment inhibited the MGMT protein expression levels in CD133+ nestin+ pituitary adenoma stem-like cells.
DSF treatment sensitizes MGMT-proficient human pituitary adenoma cells and the isolated CD133+ nestin+ stem-like cells to TMZ chemotherapy in vitro. A previous study by our group (27), it was demonstrated that 2-methoxyestradiol (2ME) increased the efficacy of TMZ in human primary culture pituitary adenoma cells in vitro and in vivo. In the present study, using the CCK8 assay on pituitary adenoma in primary culture, the sensitizing effect of DSF of pituitary adenoma cells from MGMT-negative and MGMT-positive pituitary adenomas, respectively, to TMZ treatment was assessed. Saline, TMZ (100 $\mu \mathrm{mol} / \mathrm{l})$, DSF $(25 \mu \mathrm{mol} / \mathrm{l})$ or TMZ plus DSF was added to the primary cultural human pituitary adenoma cells for $24 \mathrm{~h}$. As shown in Fig. 3A, the CCK8 assay showed that the relative proliferation ratio of strongly MGMT-positive cells in the TMZ, DSF and TMZ plus DSF groups was $0.73 \pm 0.07,1.02 \pm 0.16$ and $0.44 \pm 0.16$, respectively (TMZ vs. TMZ/DSF; $\mathrm{P}=0.025$ ), while the relative proliferation ratio of MGMT-negative cells in the TMZ, DSF and TMZ plus DSF groups was $0.67 \pm 0.09,1.10 \pm 0.11$ and $0.59 \pm 0.12$, respectively (TMZ vs. TMZ/DSF; $\mathrm{P}=0.405$ ) (Fig. 3B). These results indicated that DSF significantly enhanced the sensitivity of MGMT-positive, but not MGMTnegative cells, to TMZ.

The present study also determined this effect on pituitary adenoma stem like cells. Saline, TMZ (100 $\mu \mathrm{mol} / \mathrm{l})$, DSF (25 $\mu \mathrm{mol} / \mathrm{l})$ or TMZ plus DSF was added to the isolated CD133+ nestin+ human pituitary adenoma stem-like cells in primary culture for $24 \mathrm{~h}$. As expected, the CCK8 assay showed that the relative proliferation ratio of isolated CD133+ nestin+ human pituitary adenoma stem-like cells in the TMZ, DSF and TMZ plus DSF groups was $0.94 \pm 0.05$, $0.98 \pm 0.08$ and $0.75 \pm 0.13$, respectively (TMZ vs. TMZ/DSF; $\mathrm{P}=0.0425$ ) (Fig. 3C), indicating that DSF sensitized the isolated CD133+ nestin+ human pituitary adenoma stem-like cells to TMZ.

DSF sensitizes MGMT-proficient human pituitary adenoma xenografts to TMZ chemotherapy in vivo. To translate the in vitro findings of the present study into murine models of pituitary adenoma, the TMZ sensitization efficacy of DSF was evaluated in xenografts of MGMT-positive human pituitary adenoma cells from primary culture in athymic nude mice. As described above, once the subcutaneous xenografts formed, the mice were treated with saline, TMZ ( $3 \mathrm{mg} / \mathrm{kg}$ ), DSF (50 $\mathrm{mg} / \mathrm{kg}$ ) or TMZ plus DSF for five days. BrdU, TUNEL and MGMT staining were then performed. Although no significant difference in tumor size was identified between the different groups, the ratio of apoptotic cells in the control, TMZ, DSF and TMZ plus DSF groups was $0.31 \pm 0.11,1.32 \pm 0.13,0.35 \pm 0.14$ and $3.04 \pm 0.93 \%$, respectively (TMZ vs. TMZ/DSF; $\mathrm{P}=0.0380$ ). The ratio of BrdU-stained cells in the TMZ, DSF and TMZ plus DSF groups was $10.80 \pm 1.21,5.33 \pm 1.27,11.55 \pm 1.14$ and $2.30 \pm 1.03 \%$, respectively (TMZ vs. TMZ/DSF; $\mathrm{P}=0.0739$ ). The TMZ plus DSF group displayed the lowest BrdU staining levels and highest TUNEL staining levels (Fig. 4). Compared with those in the other groups, the MGMT expression levels were downregulated in the DSF-treated groups (Fig. 4E). These results suggested that DSF sensitized pituitary adenoma cells to TMZ in vivo. 

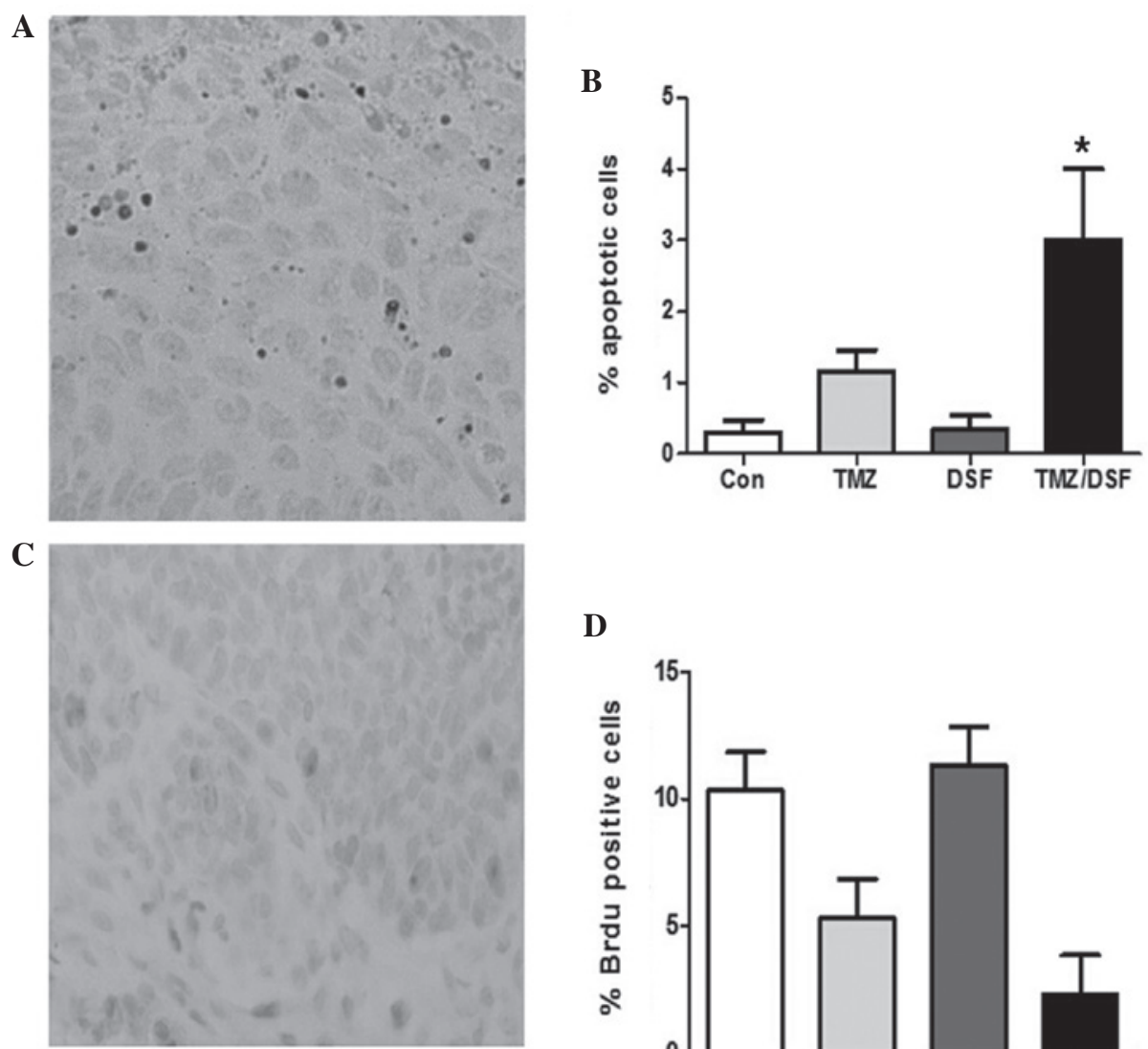

D

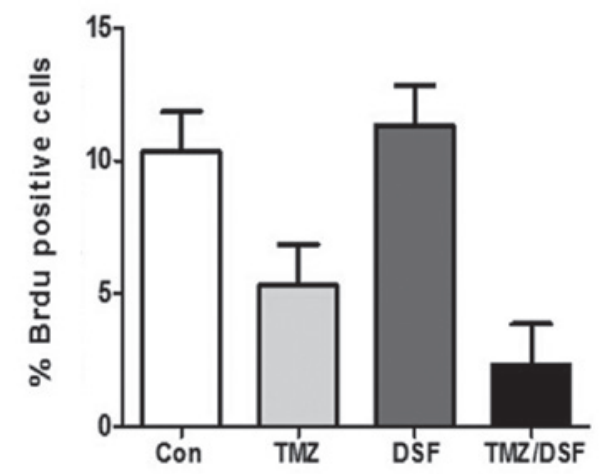

\section{E Con TMZ DSF TMZ/DSF}

MGMT

GAPDH

Figure 4. (A) Representive imaging of terminal deoxynucleotidyl transferase dUTP nick end labeling staining in human pituitary adenoma cell xenografts treated with TMZ + DSF. (B) DSF facilitates the TMZ-induced apoptosis of tumors in vivo. (C) Representative imaging of Brdu immunoreactivity in human pituitary adenoma cell xenografts treated with TMZ + DSF. (D) DSF facilitates the TMZ-induced anti-proliferative effect on tumors in vivo. (E) Western blot of MGMT protein expression in human pituitary adenoma cell xenografts in the control group and those treated with TMZ, DSF and TMZ + DSF, respectively. GAPDH served as the loading control. Magnification, $\mathrm{x} 400$. Values are expressed as the mean \pm standard deviation from three independent experiments; ${ }^{*} \mathrm{P}<0.05$, vs. TMZ group. Con, control; MGMT, O6-methylguanine-DNA methyltransferase; DSF, disulfiram; TMZ, temozolomide; BrdU, bromodeoxyuridine.

DSF treatment facilitates TMZ-induced mitotic catastrophe and apoptosis. Apoptosis, mitotic catastrophe and cell cycle arrest are known to be the main underlying mechanisms of the anti-tumor effect of TMZ (28). To characterize which of the above mechanisms of action of TMZ was facilitated by DSF in pituitary adenoma cells, the apoptosis, mitotic catastrophe and cell cycle arrest were determined in pituitary adenoma cells treated with TMZ (100 $\mu \mathrm{mol} / \mathrm{l}), \mathrm{DSF}(25 \mu \mathrm{mol} / \mathrm{l}), \mathrm{TMZ}$ plus DSF or saline (control) for $24 \mathrm{~h}$. As shown in Fig. 5, the ratio of apoptotic cells in the control, TMZ, DSF and TMZ plus DSF groups was $0.29 \pm 0.09,0.81 \pm 0.23,0.31 \pm 0.10$ and $1.64 \pm 0.16 \%$, respectively (TMZ vs. TMZ/DSF; $\mathrm{P}=0.0427$ ), which showed that the pituitary adenoma cells treated with DSF plus TMZ displayed an increased apoptotic cell population compared with cells exposed to TMZ alone. As expected, the ratio of cells in mitotic catastrophe, defined as the presence of $\geq 2$ nuclear lobes within a single cell (29), in the control, TMZ, DSF and TMZ plus DSF groups was $5.35 \pm 1.11,12.91 \pm 2.21,5.65 \pm 1.06$ and $18.27 \pm 2.34 \%$, respectively (TMZ vs. TMZ/DSF; $\mathrm{P}=0.0560$; non significant). Compared to cells treated with TMZ alone, increased mitotic catastrophe was observed in the DSF plus TMZ-treated cells (Fig. 5E). However, in contrast to what was expected, no significant changes in the cell cycle were detected in any of the groups (data not shown). As the saline group displayed no changes in the cell cycle distribution compared with that in the other groups in the present study, which was not in line with the results of previous studies $(28,30)$, this may suggest that the dose of the drugs used in the present study may not have been sufficient to affect the cell cycle; furthermore, the diversity of the primarily cultured cells may also have contributed to this inconsistency. 
A

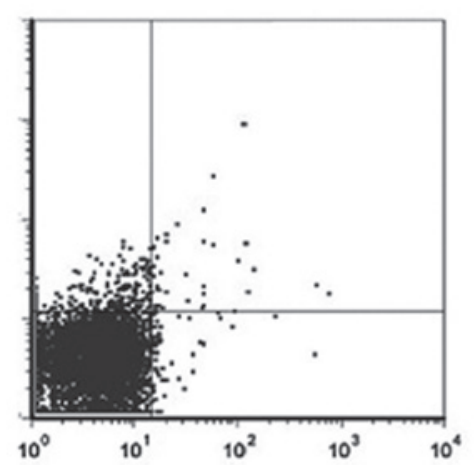

C

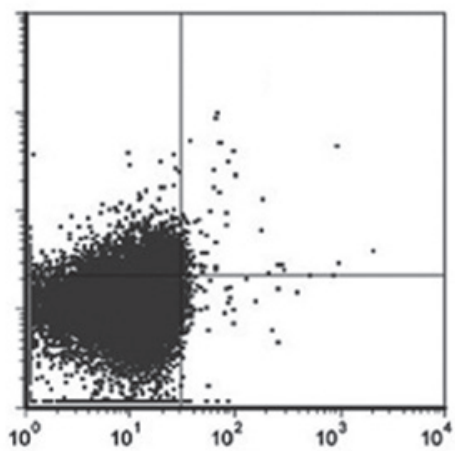

B

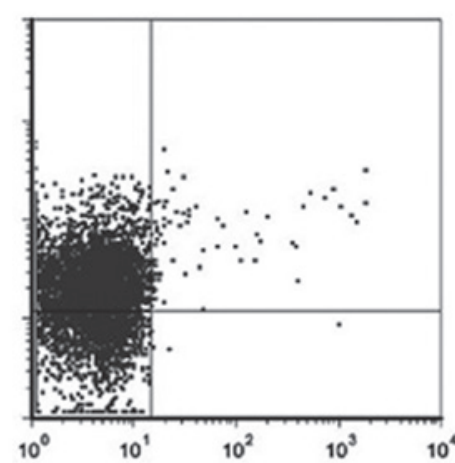

D

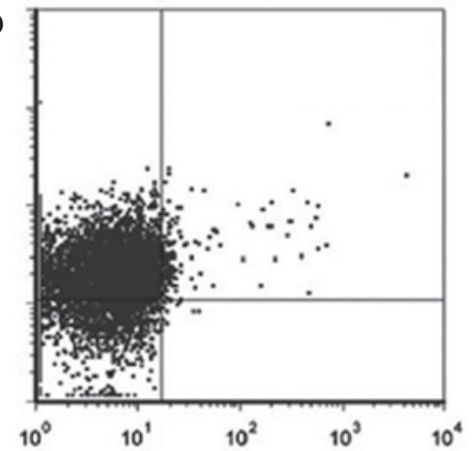

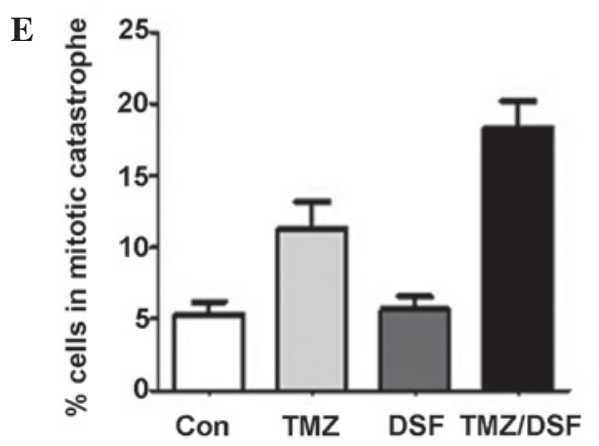

Figure 5. DSF promotes TMZ-induced apoptosis as shown by flow cytometric analysis. Representative images of cell apoptosis analysis of cells in (A) the control, (B) TMZ, (C) DSF and (D) TMZ + DSF groups. The human pituitary adenoma cells were treated with DSF or/and TMZ for $24 \mathrm{~h}$. Subsequently, cells were harvested for analysis of apoptosis. (E) DSF promotes TMZ-induced mitotic catastrophe. The human pituitary adenoma cells were treated with DSF and/or TMZ for $24 \mathrm{~h}$. Cells were fixed with cold methanol $\left(4^{\circ} \mathrm{C}\right)$ and stained with 4'-6-diamidino-2-phenylindole for mitotic catastrophe. Nuclear fragmentation (defined as the presence $\geq 2$ distinct lobes within a single cell) was evaluated in 200 cells per treatment experiment. Values are expressed as the mean \pm standard deviation from three independent experiments. DSF, disulfiram; TMZ, temozolomide; Con, control.

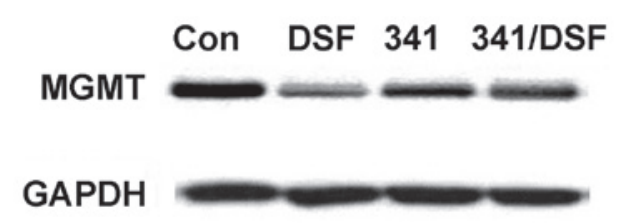

Figure 6. Western blot analysis of MGMT protein expression in human pituitary adenoma cell xenografts in the control group and those treated with DSF, PS-341 and PS-341 + DSF, respectively. GAPDH served as the loading control. Con, control; MGMT, O6-methylguanine-DNA methyltransferase; DSF, disulfiram; 341, proteasome inhibitor PS-341.

In spite of this inconsistency, the results still indicate that DSF enhances the cytotoxicity of TMZ by increasing the occurrence of mitotic catastrophe and induction of apoptosis. The results of the present study neither confirm nor deny the involvement of cell cycle arrest in DSF-facilitated TMZ toxicity.

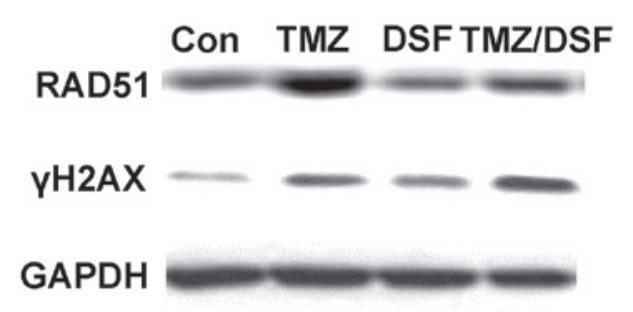

Figure 7. Western blot analysis of the RAD51 and $\gamma \mathrm{H} 2 \mathrm{AX}$ protein levels in human pituitary adenoma cell xenografts in the control group and those treated with TMZ, DSF and TMZ + DSF, respectively. GAPDH served as the loading control. Con, control; TMZ, temozolomide.

Pre-treatment with the proteasome inhibitor PS-341 abrogates the inhibition of MGMT by DSF in vitro. The effect of DSF on MGMT has been reported to be eliminated through inhibition of the ubiquitin (ub)-proteasomal route (20,31-34). To determine 
whether the enhancement of MGMT by DSF observed in the present study proceeded via the ubiquitin-proteasomal route, the proteasome inhibitor PS-341 was used. In accordance with a previous study by Paranjpe et al (20), the pituitary adenoma cells were pre-treated with PS-341 $(10 \mu \mathrm{mol} / \mathrm{l})$ for $6 \mathrm{~h}$, followed by $50 \mu \mathrm{mol} / 1 \mathrm{DSF}$ for $12 \mathrm{~h}$ in the present study. Subsequent western blot analysis showed that DSF inhibited MGMT protein expression, while PS-341 pre-treatment attenuated the DSF-induced loss of MGMT protein expression (Fig. 6). These results suggested that the interference of DSF with MGMT expression proceeds via the ub-proteasomal route.

DSF treatment inhibits the repair of TMZ-induced DNA double strand breaks (DSBS). It is known that MGMT is a unique anti-mutagenic DNA repair protein, which transfers alkyl groups to the active site of the cysteine residue as part of the DNA damage response (18,35-37). It was hypothesized that DSF inactivated the MGMT protein and inhibited the repair function of MGMT, which in turn caused an accumulation of DNA double strand breaks induced by TMZ, ultimately resulting in cell death. To confirm this mechanism, pituitary adenoma cells were treated with TMZ (100 $\mu \mathrm{mol} / \mathrm{l})$, DSF (25 $\mu \mathrm{mol} / \mathrm{l})$, TMZ plus DSF $(25 \mu \mathrm{mol} / \mathrm{l})$ or saline (control) for $24 \mathrm{~h}$. As phosphorylation of histone $\mathrm{H} 2 \mathrm{Ax}$, resulting in $\gamma \mathrm{H} 2 \mathrm{AX}$, is correlated with DSBs, and RAD51 is a protein marker associated with DNA damage repair (5,38-40), the $\gamma \mathrm{H} 2 \mathrm{AX}$ and RAD51 levels were examined by western blot analysis. As expected, the results revealed that, compared to the TMZ-treated cells, DSF plus TMZ treatment resulted in increased $\gamma \mathrm{H} 2 \mathrm{AX}$ and decreased RAD51 levels (Fig. 7). These results indicated that the reduction of MGMT levels by DSF inhibits the MGMT-mediated repair of TMZ-induced DSBs.

\section{Discussion}

TMZ, an orally administered alkylating agent, was the first chemotherapeutic agent showing anti-tumor activity against aggressive pituitary adenomas $(11,12,16)$. TMZ has been widely used as salvage therapy against aggressive pituitary adenomas resistant to conventional treatment, including surgery, dopamine agonists, somatostatin analogues and radiotherapy $(7,11-14,16,17,35,41,42)$. A previous study by our group confirmed that the DNA repair protein MGMT in human pituitary adenomas is closely associated with the tumor resistance to TMZ (19). DSF, which has been approved by the Food and Drug Administration for the treatment of alcoholism since 1951, was recently reported to inhibit MGMT protein expression and sensitize glioblastoma cells to TMZ in vitro and in vivo (20). Compared to other potential MGMT inhibitors, DSF has numerous advantages: Oral administration, the ability to cross the blood-brain barrier as well as established pharmacokinetics and drug safety $(31-34,43,44)$, all of which imply patient compliance and feasibility of fast clinical application. Based on these features, the present study hypothesizes that DSF may be used to inhibit MGMT activity in human pituitary adenomas in the clinic. In this case DSF has the potential to be used as an adjuvant therapy to chemotherapy with TMZ. This is likely to improve the efficacy of the treatment of aggressive pituitary adenomas resistant to conventional treatment, which is of key importance for patients with these tumors, as they are usually treated with TMZ as the last line therapy in clinical practice.

As there is no well-established human pituitary adenoma cell line $(45,46)$, the present study established primary cultures of human pituitary adenoma cells from tumor samples. It was found that DSF inhibited MGMT expression in these human pituitary adenoma cells in primary culture. It is known that mitotic catastrophe, apoptosis and G2/M cell cycle arrest are the main anti-tumor mechanisms of TMZ $(28,30)$. In the present study, compared to TMZ alone, DSF plus TMZ treatment resulted in increased occurrence of mitotic catastrophe and apoptosis in human pituitary adenoma cells in primary culture. Treatment with DSF plus TMZ increased the levels of $\gamma \mathrm{H} 2 \mathrm{AX}$ while decreasing protein expression levels of RAD51 and MGMT, indicating that DSF inhibited the DNA repair ability of MGMT protein in pituitary adenoma cells. However, treatment with DSF alone had no significant anti-tumor effects on pituitary adenoma cells. All these results suggested that DSF is able to improve the anti-tumor efficacy of TMZ in human pituitary adenoma cells in primary culture.

It is known that classic chemotherapeutic agents can only eliminate highly differentiated and rapidly dividing tumor cells, while less differentiated and slower proliferating tumor stem cells are predisposed to be spared by chemotherapeutic agents (47-49). Due to this premise, classical chemotherapeutic agents are likely fail to obtain successful long-term disease remission (47-49). Recently, putative pituitary adenoma stem-like cells highly expressing nestin and CD133 were obtained from human pituitary adenomas; these cells also displayed increased resistance to chemotherapeutic drugs (21). In reference to the abovementioned study (21), the present study also isolated seemingly pituitary adenoma stem-like cells using CD133- and nestin-specific magnetic microbeads. The isolated cells were shown to be able to generate spheres, differentiate into normal pituitary adenoma cells and form tumor xenografts in the right hemisphere of nude mice, indicating the self-renewal ability, multilineage differentiation and in vivo tumorigenicity of these cells. The present study also found that DSF can inhibit the expression of MGMT and sensitize pituitary adenoma stem like cells to TMZ. However, in the present study, numerous attempts to isolate nestin- and CD133-positive cells failed. These failures may be due to the rarity of the aggressive MGMT-positive pituitary adenoma tissue in the clinic, immaturity of the primary culture and the stem cell isolation technology; associated experiments are still ongoing in our laboratory.

It is known that DSF inactivate MGMT activity through eliminating MGMT protein via the ub-proteolysis pathway $(20,31-34,43,44)$. The mode of elimination of DSF-modified MGMT protein in human pituitary adenoma cells was further investigated in the present study. In line with a previous study (20), the proteasome inhibitor PS-341 pretreatment attenuated the DSF induced MGMT reduction. These results present evidence that DSF can eliminate the MGMT protein through the ub-proteolysis pathway in pituitary adenomas.

Limitations of the present study include the fact that the cells were derived from a small number of human pituitary adenomas, and more human pituitary adenomas are required to be analyzed in future studies. The diversity in primary 
cultured human pituitary adenoma cells also undermined the significance of the results of the present study. A mature human-derived pituitary cell line should be developed to better evaluate the effects of therapeutic agents in human pituitary adenomas. Due to the low abundance of human pituitary adenoma tissues as well as the requirement for development of primary culture and stem cell isolation technology, the isolation for human pituitary adenoma stem-like cells is currently difficult. During the establishment of cultures, a large quantity of valuable but limited human aggressive pituitary adenoma tissues was wasted in the isolation process. As the numbers of acquired human pituitary adenoma stem-like cells are generally low, only a small number of studies using human pituitary adenoma stem-like cells have been performed to date. Further studies are required to improve the primary culture and stem cell isolation technology. Finally, due to the immature cellar implantation technology and the rarity of the primarily cultured cells, the tumor formation rate was low. The present study only verified the tumor initiation ability of the isolated stem-like cells in the right cerebral hemisphere of nude mice. The cells were implanted subcutaneously into the lower rear flank of nude mice in the other in vivo experiments, while intracranial implantation of cells in the right cerebral hemisphere of nude mice may have been able to improve the significance of the in vivo experiment in the present study, by better mimicking pathological and physiological behavior of pituitary adenoma cells. Further studies using the intracranial implantation of pituitary adenoma cells are required.

In spite of these limitations, the present study presented the first evidence that DSF can inhibit MGMT levels and sensitize human pituitary adenoma cells and stem like cells to the anti-tumor drug TMZ. Although these results remain to be translated into clinical applications with caution, the sensitizing effect of DSF in the setting of TMZ therapy may offer added benefit. Further characterization of the benefit-to-risk profile of TMZ plus DSF in patients with aggressive pituitary adenomas refractory to conventional treatments is required to justify this combined treatment.

\section{Acknowledgements}

The current study was supported by a joint training program foundation for talents in Henan Province, China (grant no. U1404822).

\section{References}

1. Beckers A: Higher prevalence of clinically relevant pituitary adenomas confirmed. Clin Endocrinol (Oxf) 72: 290-291, 2010.

2. Tamer G, Telci A, Mert M, Uzum AK, Aral F, Tanakol R, Yarman S, Boztepe H, Colak N and Alagöl F: Prevalence of pituitary adenomas in macroprolactinemic patients may be higher than it is presumed. Endocrine 41: 138-143, 2012.

3. Karavitaki N: Prevalence and incidence of pituitary adenomas. Ann Endocrinol (Paris) 73: 79-80, 2012.

4. Ezzat S, Asa SL, Couldwell WT, Barr CE, Dodge WE, Vance ML and McCutcheon IE: The prevalence of pituitary adenomas: a systematic review. Cancer 101: 613-619, 2004.

5. Selman WR, Laws ER Jr, Scheithauer BW and Carpenter SM: The occurrence of dural invasion in pituitary adenomas. J Neurosurg 64: 402-407, 1986.

6. Scheithauer BW, Kovacs KT, Laws ER Jr and Randall RV: Pathology of invasive pituitary tumors with special reference to functional classification. J Neurosurg 65: 733-744, 1986.
7. Zemmoura I, Wierinckx A, Vasiljevic A, Jan M, Trouillas J and François P: Aggressive and malignant prolactin pituitary tumors: pathological diagnosis and patient management. Pituitary 16: 515-522, 2013.

8. Levy A: Pituitary disease: presentation, diagnosis and management. J Neurol Neurosurg Psychiatry 75 (Suppl 3): iii47-52, 2004.

9. Buchfelder M: Management of aggressive pituitary adenomas: current treatment strategies. Pituitary 12: 256-260, 2009.

10. Maïza JC and Caron P: Pituitary carcinomas and aggressive adenomas: an overview and new therapeutic options. Ann Endocrinol (Paris) 70 (Suppl 1): 12-19, 2009 (In French).

11. Syro LV, Uribe H, Penagos LC, Ortiz LD, Fadul CE, Horvath E and Kovacs K: Antitumour effects of temozolomide in a man with a large, invasive prolactin-producing pituitary neoplasm. Clin Endocrinol (Oxf) 65: 552-553, 2006.

12. Neff LM, Weil M, Cole A, Hedges TR, Shucart W, Lawrence D, Zhu JJ, Tischler AS and Lechan RM: Temozolomide in the treatment of an invasive prolactinoma resistant to dopamine agonists. Pituitary 10: 81-86, 2007.

13. Mohammed S, Kovacs K, Mason W, Smyth H and Cusimano MD: Use of temozolomide in aggressive pituitary tumors: case report. Neurosurgery 64: E773-774, discussion E774, 2009.

14. Kaltsas GA, Mukherjee JJ, Plowman PN, Monson JP, Grossman AB and Besser GM: The role of cytotoxic chemotherapy in the management of aggressive and malignant pituitary tumors. J Clin Endocrinol Metab 83: 4233-4238, 1998.

15. Zhou PZ, Ma WC, Wang XJ, Cheng SW and Jiang S: Isolation and preliminary identification of stem-like cells in pituitary adenoma. Sichuan Da Xue Xue Bao Yi Xue Ban 44: 466-469, 2013 (In Chinese).

16. Raverot G, Sturm N and de Fraipont F: Temozolomide treatment in aggressive pituitary tumors and pituitary carcinomas: a French multicenter experience. J Clin Endocrinol Metab 95: 4592-4599, 2010.

17. Bush ZM, Longtine JA, Cunningham T, Schiff D, Jane JA Jr, Vance ML, Thorner MO, Laws ER Jr and Lopes MB: Temozolomide treatment for aggressive pituitary tumors: correlation of clinical outcome with $\mathrm{O}$ (6)-methylguanine methyltransferase (MGMT) promoter methylation and expression. J Clin Endocrinol Metab 95: E280-290, 2010.

18. Kovacs K, Scheithauer BW, Lombardero M, McLendon RE, Syro LV, Uribe H, Ortiz LD and Penagos LC: MGMT immunoexpression predicts responsiveness of pituitary tumors to temozolomide therapy. Acta Neuropathol 115: 261-262, 2008.

19. Chen W, Xiao Z, Zhao Y, Huang L and Du G: HIF-1 $\alpha$ inhibition sensitizes pituitary adenoma cells to temozolomide by regulating MGMT expression. Oncol Rep 30: 2495-2501, 2013.

20. Paranjpe A, Zhang R, Ali-Osman F, Bobustuc GC and Srivenugopal KS: Disulfiram is a direct and potent inhibitor of human O6-methylguanine-DNA methyltransferase (MGMT) in brain tumor cells and mouse brain and markedly increases the alkylating DNA damage. Carcinogenesis 35: 692-702, 2014.

21. Xu Q, Yuan X, Tunici P and et al: Isolation of tumour stem-like cells from benign tumours. Br J Cancer 101: 303-311, 2009.

22. Wang X, Ma Z, Xiao Z, Liu H, Dou Z, Feng X and Shi H: Chk1 knockdown confers radiosensitization in prostate cancer stem cells. Oncol Rep 28: 2247-2254, 2012.

23. Xiao Z, Liu Q, Mao F, Wu J and Lei T: TNF- $\alpha$-induced VEGF and MMP-9 expression promotes hemorrhagic transformation in pituitary adenomas. Int J Mol Sci 12: 4165-4179, 2011.

24. Xiao Z, Liu Q, Zhao B, Wu J and Lei T: Hypoxia induces hemorrhagic transformation in pituitary adenomas via the HIF-1 $\alpha$ signaling pathway. Oncol Rep 26: 1457-1464, 2011

25. Yang J, Xiao Z, Li T, Gu X and Fan B: Erythropoietin promotes the growth of pituitary adenomas by enhancing angiogenesis. Int J Oncol 40: 1230-1237, 2012.

26. Wang X, Ma Z, Xiao Z, Liu H, Dou Z, Feng X and Shi H: Chk1 knockdown confers radiosensitization in prostate cancer stem cells. Oncol Rep 28: 2247-2254, 2012.

27. Chen W, Xiao Z, Zhao Y, Huang L and Du G: HIF-1 $\alpha$ inhibition sensitizes pituitary adenoma cells to temozolomide by regulating MGMT expression. Oncol Rep 30: 2495-2501, 2013.

28. O'Reilly SM, Newlands ES, Glaser MG, et al: Temozolomide: a new oral cytotoxic chemotherapeutic agent with promising activity against primary brain tumours. Eur J Cancer 29A: 940-942, 1993.

29. Castedo M, Perfettini JL, Roumier T, Andreau K, Medema R and Kroemer G: Cell death by mitotic catastrophe: a molecular definition. Oncogene 23: 2825-2837, 2004. 
30. Bocangel DB, Finkelstein S, Schold SC, Bhakat KK, Mitra S and Kokkinakis DM: Multifaceted resistance of gliomas to temozolomide. Clin Cancer Res 8: 2725-2734, 2002.

31. Cen D, Brayton D, Shahandeh B, Meyskens FL Jr and Farmer PJ: Disulfiram facilitates intracellular $\mathrm{Cu}$ uptake and induces apoptosis in human melanoma cells. J Med Chem 47: 6914-6920, 2004.

32. Cvek B: Targeting malignancies with disulfiram (Antabuse): multidrug resistance, angiogenesis and proteasome. Curr Cancer Drug Targets 11: 332-337, 2011.

33. Triscott J, Lee C, Hu K, et al: Disulfiram, a drug widely used to control alcoholism, suppresses the self-renewal of glioblastoma and over-rides resistance to temozolomide. Oncotarget 3 : $1112-1123,2012$

34. Johansson B: A review of the pharmacokinetics and pharmacodynamics of disulfiram and its metabolites. Acta Psychiatr Scand Suppl 369: 15-26, 1992.

35. McCormack AI, McDonald KL, Gill AJ, et al: Low O6-methylguanine-DNA methyltransferase (MGMT) expression and response to temozolomide in aggressive pituitary tumours. Clin Endocrinol (Oxf) 71: 226-233, 2009.

36. Gerson SL: MGMT: its role in cancer aetiology and cancer therapeutics. Nat Rev Cancer 4: 296-307, 2004.

37. Widhalm G, Wolfsberger S, Preusser M, Woehrer A, Kotter MR Czech T, Marosi C and Knosp E: O (6)-methylguanine DNA methyltransferase immunoexpression in nonfunctioning pituitary adenomas: are progressive tumors potential candidates for temozolomide treatment. Cancer 115: 1070-1080, 2009.

38. Syljuåasen RG, Sørensen CS, Hansen LT, Fugger K, Lundin C, Johansson F, Helleday T, Sehested M, Lukas J and Bartek J: Inhibition of human Chk1 causes increased initiation of DNA replication, phosphorylation of ATR targets and DNA breakage. Mol Cell Biol 25: 3553-3562, 2005.

39. Sorensen CS, Hansen LT, Dziegielewski J, Syljuåsen RG, Lundin C, Bartek J and Helleday T: The cell-cycle checkpoint kinase Chk1 is required for mammalian homologous recombination repair. Nat Cell Biol 7: 195-201, 2005.
40. Bahassi EM, Ovesen JL, Riesenberg AL, Bernstein WZ, Hasty PE and Stambrook PJ: The checkpoint kinases Chk1 and Chk2 regulate the functional associations between hBRCA2 and Rad51 in response to DNA damage. Oncogene 27: 3977-3985, 2008.

41. Beck-Peccoz P, Lania A, Beckers A, Chatterjee K and Wemeau JL: 2013 European thyroid association guidelines for the diagnosis and treatment of thyrotropin-secreting pituitary tumors. Eur Thyroid J 2: 76-82, 2013, 2013.

42. Kovacs K, Horvath E, Syro LV, Uribe H, Penagos LC, Ortiz LD and Fadul CE: Temozolomide therapy in a man with an aggressive prolactin-secreting pituitary neoplasm: Morphological findings. Hum Pathol 38: 185-189, 2007.

43. Wickström M, Danielsson K, Rickardson L, Gullbo J, Nygren P, Isaksson A, Larsson R and Lövborg H: Pharmacological profiling of disulfiram using human tumor cell lines and human tumor cells from patients. Biochem Pharmacol 73: 25-33, 2007.

44. Chen D, Cui QC, Yang H and Dou QP: Disulfiram, a clinically used anti-alcoholism drug and copper-binding agent, induces apoptotic cell death in breast cancer cultures and xenografts via inhibition of the proteasome activity. Cancer Res 66: 10425-10433, 2006.

45. Rizzoti K: Adult pituitary progenitors/stem cells: from in vitro characterization to in vivo function. Eur J Neurosci 32: 2053-2062, 2010

46. Florio T: Adult pituitary stem cells: from pituitary plasticity to adenoma development. Neuroendocrinology 94: 265-277, 2011.

47. Visvader JE and Lindeman GJ: Cancer stem cells in solid tumours: accumulating evidence and unresolved questions. Nat Rev Cancer 8: 755-768, 2008.

48. Eyler CE and Rich JN: Survival of the fittest: cancer stem cells in therapeutic resistance and angiogenesis. J Clin Oncol 26: 2839-2845, 2008

49. Fernandez A, Karavitaki N and Wass JA: Prevalence of pituitary adenomas: a community-based, cross-sectional study in Banbury (Oxfordshire, UK). Clin Endocrinol (Oxf) 72: 377-382, 2010 . 